\title{
Universal quantum computation and quantum error correction using discrete holonomies
}

\author{
Cornelis J. G. Mommers and Erik Sjöqvist $\odot^{*}$ \\ Department of Physics and Astronomy, Uppsala University, Box 516, Se-751 20 Uppsala, Sweden
}

(Received 1 September 2021; accepted 21 January 2022; published 7 February 2022)

\begin{abstract}
Holonomic quantum computation exploits a quantum state's nontrivial, matrix-valued geometric phase (holonomy) to perform fault-tolerant computation. Holonomies arising from systems where the Hamiltonian traces a continuous path through parameter space have been well researched. Discrete holonomies, on the other hand, where the state jumps from point to point in state space, have had little prior investigation. Using a sequence of incomplete projective measurements of the spin operator, we build an explicit approach to universal quantum computation. We show that quantum error correction codes integrate naturally in our scheme, providing a model for measurement-based quantum computation that combines the passive error resilience of holonomic quantum computation and active error correction techniques. In the limit of dense measurements we recover known continuous-path holonomies.
\end{abstract}

DOI: 10.1103/PhysRevA.105.022402

\section{INTRODUCTION}

In the circuit model of quantum computation, information is processed by using a series of quantum gates on a register of qubits. These gates are unitary transformations, which can be realized using non-Abelian geometric phases (holonomies) [1] that make them intrinsically fault tolerant [2]. Holonomic quantum computation is often studied in adiabatic systems, where the Hamiltonian traces a continuous path through parameter space. Experimental realizations of adiabatic holonomic quantum computation in a wide range of physical settings have been proposed [3-5] and implemented $[6,7]$. On the other hand, there has not been as much research into the discrete case, whereby the state jumps from point to point in state space resulting in non-Abelian holonomies [8-10].

Here, we examine holonomies arising from sequences of incomplete measurements. We demonstrate a universal set of holonomic quantum gates acting on qubits implemented by a discrete set of incomplete measurements on spin-coherent states (SCSs) [11,12]. SCSs can be created in the laboratory $[13,14]$ and have mathematically desirable properties [15]. In the limit of dense measurements our scheme reduces to wellknown continuous-path holonomies, which, for SCSs, are Abelian [16] and therefore insufficient to achieve universality. Hence, to realize truly non-Abelian holonomies acting on SCS qubits it is essential to use (discrete) sequences consisting of a finite number of measurements.

\footnotetext{
*erik.sjoqvist@physics.uu.se
}

Published by the American Physical Society under the terms of the Creative Commons Attribution 4.0 International license. Further distribution of this work must maintain attribution to the author(s) and the published article's title, journal citation, and DOI. Funded by Bibsam.
We further show that measurement-driven holonomies on SCSs can be naturally merged with active error correction techniques, such as direct implementation of the bit-flip repetition code [17] and extension into the nine-qubit Shor code [18]. In this way, our proposed scheme can be viewed as a model for measurement-based quantum computation, in the same vein as, e.g., one-way cluster state quantum computation [19] and teleportation-based quantum computation [20]. Our approach combines the passive error resilience of holonomic quantum computation and active error correction techniques, making it a promising tool for robust quantum computation.

\section{HOLONOMIC SCHEME}

\section{A. Preliminaries}

To introduce notation, we begin by briefly outlining some conceptual aspects of discrete holonomies. Suppose we have an $N$-dimensional Hilbert space. A projection onto a $K$ dimensional subspace $p_{a}$, spanned by a (nonunique) frame $\mathcal{F}_{a}=\left\{\left|a_{k}\right\rangle\right\}_{k=1}^{K}$, can be realized with a projector $P_{a}$. The overlap matrix, defined componentwise as

$$
\left(\mathcal{F}_{a} \mid \mathcal{F}_{b}\right)_{k l}=\left\langle a_{k} \mid b_{l}\right\rangle
$$

quantifies how different subspaces are connected [21]. From a cyclic sequence $C$ of $q+1$ projections ( $q$ of which are distinct) we can construct $\Gamma_{C}$, given by

$$
\Gamma_{C}=P_{1} P_{q} P_{q-1} \cdots P_{1} .
$$

$\Gamma_{C}$ can be realized by applying a sequence of $q+1$ projective filtering measurements that transforms an input state $|\psi\rangle \in p_{1}$ as

$$
|\psi\rangle \rightarrow \sum_{k, l}\left|1_{k}\right\rangle D_{k l}\left\langle 1_{l} \mid \psi\right\rangle,
$$

with $D=\left(\mathcal{F}_{1} \mid \mathcal{F}_{q}\right)\left(\mathcal{F}_{q} \mid \mathcal{F}_{q-1}\right) \cdots\left(\mathcal{F}_{2} \mid \mathcal{F}_{1}\right)$ [9]. The holonomy of $C$ is the unitary part $U_{D}=|D|^{-1} D$ of $D$, with $|D|=\sqrt{D D^{\dagger}}$ 
the positive part of $D$. In the following, we take $p_{1}$ to be the computational subspace.

Next, let us parametrize a unit vector $\mathbf{n}$ with spherical coordinates as $(\sin \theta \cos \phi, \sin \theta \sin \phi, \cos \theta)$. With $\{|j, m\rangle\}_{m=-j}^{j}$ denoting the eigenkets of the $J_{z}$ operator, the SCSs read $e^{-i \phi J_{z}} e^{-i \theta J_{y}}|j, \pm j\rangle$ (we put $\hbar=1$ from now on, and abbreviate $|j, \pm j\rangle$ to $| \pm j\rangle$ ). If $j \geqslant 1$, we can have our sequence of frames be projections onto subspaces spanned by different SCSs, viz.,

$$
\mathcal{F}_{a}\left(\theta_{a}, \phi_{a}\right)=\left\{e^{-i \phi_{a} J_{z}} e^{-i \theta_{a} J_{y}}|j, \pm j\rangle\right\}=\left\{\left| \pm j ; \mathbf{n}_{a}\right\rangle\right\}
$$

Each projective filtering measurement corresponds to the operator $P_{a}=\left|+j ; \mathbf{n}_{a}\right\rangle\left\langle+j ; \mathbf{n}_{a}|+|-j ; \mathbf{n}_{a}\right\rangle\left\langle-j ; \mathbf{n}_{a}\right|$ and represents the degenerate measurement outcome $j^{2}$ of the observable $\left(\mathbf{n}_{a} \cdot \mathbf{J}\right)^{2}$.

We can calculate the overlap matrix for the SCSs by decomposing each state into a tensor product of spin- $\frac{1}{2}$ states, $| \pm j\rangle=\left| \pm \frac{1}{2}\right\rangle^{\otimes 2 j}$. One finds nonvanishing overlap matrices [9]

$$
\left(\mathcal{F}_{a} \mid \mathcal{F}_{b}\right)=\left(\begin{array}{cc}
R_{a, b} & S_{a, b} \\
(-1)^{2 j} S_{a, b}^{*} & R_{a, b}^{*}
\end{array}\right),
$$

with

$$
\begin{aligned}
R_{a, b}= & {\left[\cos \left(\frac{\theta_{a}-\theta_{b}}{2}\right) \cos \left(\frac{\phi_{a}-\phi_{b}}{2}\right)\right.} \\
& \left.+i \cos \left(\frac{\theta_{a}+\theta_{b}}{2}\right) \sin \left(\frac{\phi_{a}-\phi_{b}}{2}\right)\right]^{2 j}, \\
S_{a, b}= & {\left[\sin \left(\frac{\theta_{a}-\theta_{b}}{2}\right) \cos \left(\frac{\phi_{a}-\phi_{b}}{2}\right)\right.} \\
& \left.-i \sin \left(\frac{\theta_{a}+\theta_{b}}{2}\right) \sin \left(\frac{\phi_{a}-\phi_{b}}{2}\right)\right]^{2 j} .
\end{aligned}
$$

To make the holonomy unambiguously associated with a quantum gate we require that the overlap matrices are unitary up to a multiplicative positive number. From now on we restrict $j$ to $j=(2 n+1) / 2, n \in \mathbb{N}$. Then all the SCSs subspaces will be fully overlapping [22]. We define our qubit as $|0\rangle=|j\rangle$ and $|1\rangle=|-j\rangle$. Our input state is $|\psi\rangle=a|j\rangle+$ $b|-j\rangle$ with $|a|^{2}+|b|^{2}=1$ and $| \pm j\rangle \in \mathcal{F}_{1}$, where $\mathcal{F}_{1}$ spans the first subspace. A $|j\rangle$ state will be composed of $(2 n+1)$ spin- $\frac{1}{2}$ constituents [23]. We can make a left polar decomposition of the overlap matrix into

$$
\left(\mathcal{F}_{a} \mid \mathcal{F}_{b}\right)=\left|\left(\mathcal{F}_{a} \mid \mathcal{F}_{b}\right)\right| U_{a, b}=\kappa_{a, b}^{-1} U_{a, b},
$$

where $\kappa_{a, b}^{-1}=\sqrt{\left|R_{a, b}\right|^{2}+\left|S_{a, b}\right|^{2}}>0$ and $U_{a, b}$ is a unique unitary matrix [24]. If we perform a sequence of $q+1$ measurements projecting onto the SCS subspaces, the final holonomy becomes

$$
U_{D}=\kappa_{1, q} \kappa_{q, q-1} \cdots \kappa_{2,1}\left(\mathcal{F}_{1} \mid \mathcal{F}_{q}\right)\left(\mathcal{F}_{q} \mid \mathcal{F}_{q-1}\right) \cdots\left(\mathcal{F}_{2} \mid \mathcal{F}_{1}\right) .
$$

\section{B. Single-qubit gates}

We consider sequences of four measurements, which is the minimum number of measurements that can yield a nontrivial holonomy [25]. The first measurement is a projection onto the same subspace as $|\psi\rangle$. This projection is the preparation of our state in the correct subspace. We then carry out two more projections onto subspaces that are different from the first subspace and each other. Finally, we project back onto the same subspace as the starting measurement. Without loss of generality we define $\left(\theta_{1}, \phi_{1}\right)=\left(\theta_{4}, \phi_{4}\right)=(0,0)$. Our sequence of measurements becomes $(0,0) \rightarrow\left(\theta_{2}, \phi_{2}\right) \rightarrow$ $\left(\theta_{3}, \phi_{3}\right) \rightarrow(0,0)$.

An astute reader may raise objections to our use of $(0,0)$ as our starting state because spherical coordinates are undefined at the poles. There, we have $e^{-i \phi J_{z}}| \pm j\rangle$ and $e^{-i \phi J_{z}} e^{-i \pi J_{y}}| \pm j\rangle$, respectively, so there is no unique eigenstate. However, we were careful to define the $(0,0)$ label to correspond to our starting and ending subspace, which is unambiguous [26]. Subsequent measurements are projections onto rotated versions of the original subspace, which is readily seen when we recall that $e^{-i \phi \mathbf{n} \cdot \mathbf{J}}=\mathcal{D}_{\mathbf{n}}(\phi)$ is the rotation operator around the direction $\mathbf{n}$.

We want to find the rotation gates, as any single-qubit operation can be decomposed exactly into $\mathcal{D}_{z}(\alpha) \mathcal{D}_{y}(\beta) \mathcal{D}_{z}(\gamma)$. Note that for the rest of this analysis any identification should be understood as an identification up to an unimportant global phase factor.

We first implement a rotation gate about the $z$ axis. We pick $\left(\theta_{2}, \phi_{2}, \theta_{3}, \phi_{3}\right)=\left(\frac{\pi}{2}, \pi, \frac{\pi}{2}, \varphi\right)$, where $\varphi \in[0,2 \pi)$, and find

$$
\begin{aligned}
\mathcal{D}_{z}(\phi)=U_{D, z} & =\frac{1}{\sqrt{\cos ^{2+4 n}\left(\frac{\varphi}{2}\right)+\sin ^{2+4 n}\left(\frac{\varphi}{2}\right)}}\left(\begin{array}{cc}
z^{*} & 0 \\
0 & z
\end{array}\right) \\
& =\left(\begin{array}{cc}
e^{-i \phi / 2} & 0 \\
0 & e^{i \phi / 2}
\end{array}\right),
\end{aligned}
$$

with

$$
z=e^{i(2 n+1) \frac{\varphi}{2}}\left[(-1)^{n} \cos ^{2 n+1}\left(\frac{\varphi}{2}\right)-i \sin ^{2 n+1}\left(\frac{\varphi}{2}\right)\right],
$$

where the final identification of Eq. (9) holds since $U_{D} \in$ $\mathrm{SU}(2)$, so $|z|=1$. The relative phase change induced by this holonomy is

$$
\phi=\arg \left(z / z^{*}\right) \in(-\pi, \pi] .
$$

Rotations about the $x$ and $y$ axes are given by

$$
\begin{aligned}
& U_{D, x}=\frac{1}{\sqrt{\cos ^{2+4 n}\left(\frac{\varphi}{2}\right)+\sin ^{2+4 n}\left(\frac{\varphi}{2}\right)}}\left(\begin{array}{cc}
\operatorname{Re}(z) & i \operatorname{Im}(z) \\
i \operatorname{Im}(z) & \operatorname{Re}(z)
\end{array}\right), \\
& U_{D, y}=\frac{1}{\sqrt{\cos ^{2+4 n}\left(\frac{\varphi}{2}\right)+\sin ^{2+4 n}\left(\frac{\varphi}{2}\right)}}\left(\begin{array}{cc}
\operatorname{Re}(z) & -\operatorname{Im}(z) \\
\operatorname{Im}(z) & \operatorname{Re}(z)
\end{array}\right) .
\end{aligned}
$$

These matrices are equivalent to the following list of angles:

$$
\begin{aligned}
U_{D, x}=\mathcal{D}_{x}(\phi):(0,0) & \rightarrow\left(\frac{\pi}{2}, \pi\right) \rightarrow\left(\varphi, \frac{\pi}{2}\right) \rightarrow(0,0) \\
U_{D, y}=\mathcal{D}_{y}(\phi):(0,0) & \rightarrow\left(\varphi, \begin{array}{l}
0 \text { if } n \text { is even } \\
\pi \text { if } n \text { is odd }
\end{array}\right) \\
& \rightarrow\left(\frac{\pi}{2}, \frac{\pi}{2}\right) \rightarrow(0,0)
\end{aligned}
$$

where $\varphi$ can be found by solving Eq. (11). 


\section{Two-qubit gate}

To construct an entangling two-qubit gate necessary for universality we make use of the auxiliary states $\left|\zeta_{ \pm}\right\rangle \in \mathcal{F}_{1}$ for the second qubit, with $\left\langle\zeta_{+} \mid \zeta_{-}\right\rangle=\xi$, in addition to the input state $|\psi\rangle$ of the first qubit. Our two-qubit gate only rotates the first qubit, keeping the subspace of the second qubit fixed. In the extended Hilbert space our new frames are $\mathcal{F}_{a}=\left\{\left| \pm j ; \mathbf{n}_{a}\right\rangle \otimes\left|\xi_{ \pm}\right\rangle\right\}$, with corresponding rank2 projection operators $P_{a}=\left|+j ; \mathbf{n}_{a}\right\rangle\left\langle+j ; \mathbf{n}_{a}|\otimes| \xi_{+}\right\rangle\left\langle\xi_{+}\right|+$ $\left|-j ; \mathbf{n}_{a}\right\rangle\left\langle-j ; \mathbf{n}_{a}|\otimes| \xi_{-}\right\rangle\left\langle\xi_{-}\right|$. The new overlap matrices are

$$
\left(\mathcal{F}_{a} \mid \mathcal{F}_{b}\right)=\left(\begin{array}{cc}
R_{a, b} & \xi S_{a, b} \\
-\xi^{*} S_{a, b}^{*} & R_{a, b}^{*}
\end{array}\right)=\sqrt{\left|R_{a, b}\right|^{2}+\left|\xi S_{a, b}\right|^{2}} \tilde{U}_{a, b}
$$

If $\left|\zeta_{+}\right\rangle=\left|\zeta_{-}\right\rangle$, then $\xi=1$ and we recover the single-qubit gates acting on the first qubit. If $\xi=0$, then

$$
\left(\mathcal{F}_{a} \mid \mathcal{F}_{b}\right)=\left(\begin{array}{cc}
R_{a, b} & 0 \\
0 & R_{a, b}^{*}
\end{array}\right)=\left|R_{a, b}\right|\left(\begin{array}{cc}
e^{i \phi_{a, b}} & 0 \\
0 & e^{-i \phi_{a, b}}
\end{array}\right),
$$

which is a phase gate inducing a phase shift $\Phi=\phi_{1, q}+$ $\phi_{q, q-1}+\cdots+\phi_{2,1}$. If we have an input state $|\psi\rangle \otimes|\tilde{\psi}\rangle$, where $|\tilde{\psi}\rangle=c|j\rangle+d|-j\rangle$, with $|c|^{2}+|d|^{2}=1$, then the action of the gate is

$$
\begin{aligned}
& (a|j\rangle+b|-j\rangle) \otimes|\tilde{\psi}\rangle \mapsto a e^{i \Phi}\left\langle\zeta_{+} \mid \tilde{\psi}\right\rangle|j\rangle\left|\xi_{+}\right\rangle \\
& \quad+b e^{-i \Phi}\left\langle\zeta_{-} \mid \tilde{\psi}\right\rangle|-j\rangle\left|\xi_{-}\right\rangle .
\end{aligned}
$$

This gate can entangle. For instance, if we pick $\left|\zeta_{ \pm}\right\rangle=| \pm j\rangle$ the concurrence [27] will be proportional to $|a b c d|$, which is nonzero in general. The fact that we can construct all singlequbit gates from the rotation gates and that we can create an entangling two-qubit gate implies universality [28].

\section{Example}

It is instructive to work out the discrete set of single-qubit Clifford $+T$ gates [29] (the $S, H$, and $T$ gates) for $j=\frac{3}{2}$, which are sufficient for universal quantum computation when supplemented by an entangling two-qubit gate. The procedure for higher- $j$ gates is identical. Plugging $n=1$ into Eq. (9) gives us

$$
U_{D, z}^{\left(j=\frac{3}{2}\right)}=\frac{1}{\sqrt{10+6 \cos (2 \varphi)}}\left(\begin{array}{cc}
1+3 e^{-2 i \varphi} & 0 \\
0 & 1+3 e^{2 i \varphi}
\end{array}\right)
$$

Therefore,

$$
\phi=\arg \left(\frac{1+3 e^{-2 i \varphi}}{1+3 e^{2 i \varphi}}\right) .
$$

A plot of Eq. (18) is shown in Fig. 1. Note that $\phi$ is multivalued. However, each intersection point yields the same holonomy. For the $T$ and $S$ gates we want to find the intersection point where $\phi$ is $\frac{\pi}{4}$ and $\frac{\pi}{2}$, respectively. $H$ can be decomposed into a $\frac{\pi}{2}$ rotation around the $y$ axis and a $\pi$ rotation around the $z$ axis. We find that the gates can be realized using the following sequence of

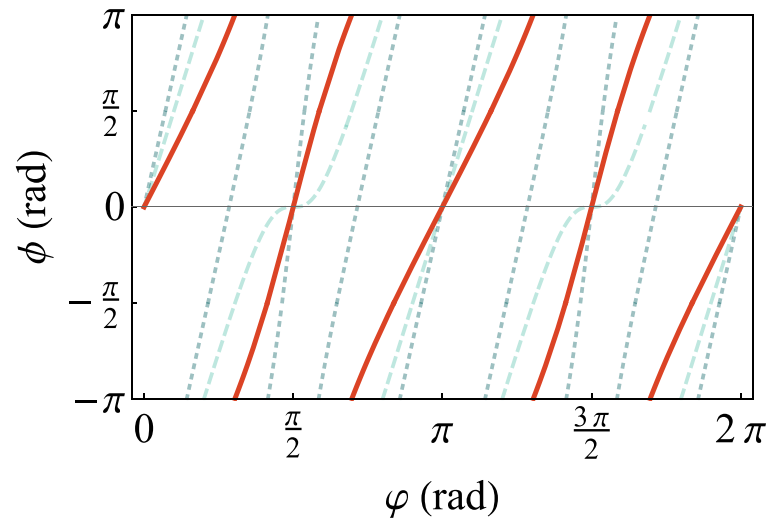

FIG. 1. The relative phase $\phi$ as a function of the subspace rotation angle $\varphi$ for $j=\frac{3}{2}$ (solid), $j=\frac{5}{2}$ (dashed), and $j=\frac{7}{2}$ (dotted).

measurements:

$$
\begin{aligned}
T:(0,0) & \rightarrow\left(\frac{\pi}{2}, \pi\right) \rightarrow\left(\frac{\pi}{2}, \phi_{T}\right) \rightarrow(0,0), \\
S:(0,0) & \rightarrow\left(\frac{\pi}{2}, \pi\right) \rightarrow\left(\frac{\pi}{2}, \phi_{S}\right) \rightarrow(0,0), \\
H:(0,0) & \rightarrow\left(\phi_{S}, \pi\right) \rightarrow\left(\frac{\pi}{2}, \frac{\pi}{2}\right) \rightarrow(0,0) \rightarrow\left(\frac{\pi}{2}, \pi\right) \\
& \rightarrow\left(\frac{\pi}{2}, \arctan \sqrt{2} \approx 0.955\right) \rightarrow(0,0),
\end{aligned}
$$

with $\phi_{T}=2 \operatorname{arcsec}\left(2 \sqrt{\frac{6}{\sqrt{6(\sqrt{2}-\sqrt{36 \sqrt{2}+70}+10)}+12}}\right) \approx 1.44$ and $\phi_{S}=\arctan \left(\frac{3+\sqrt{17}}{2}\right) \approx 1.30$. Note that these sequences are not unique. For example, for the $H$ gate we can replace the fifth pair of angles by $\left(\frac{\pi}{2}, 0\right)$.

\section{GATE PERFORMANCE AND ERROR CORRECTION CODES}

Having constructed the formalism to describe holonomic quantum gates, we now turn to their performance. For each projection onto a subspace $p$ there is a chance our state collapses onto the orthogonal subspace $p^{\perp}$. So, we can quantify the gates' performance by looking at the total transition amplitude into the intended subspace. We focus on the rotation gates since they form the basis for all single-qubit gates. Furthermore, due to symmetry, we only need to look at rotations around the $z$ axis. It turns out that the transition amplitude $T_{\mathcal{C}}$ is independent of the input state and is given by

$$
T_{C}=4^{-2 n}\left[\cos ^{2+4 n}\left(\frac{\varphi}{2}\right)+\sin ^{2+4 n}\left(\frac{\varphi}{2}\right)\right] .
$$

For $j=\frac{3}{2}$ the maximum value of the transition amplitude is $\frac{1}{16} \approx 0.063$, while the minimum value is $\frac{1}{64} \approx 0.016$. As we increase $j$ (and so the number of spin- $\frac{1}{2}$ constituents), the transition amplitude decreases exponentially. Hence, we should pick $j$ to be as low as possible to have a feasible gate.

For most practical purposes, even the maximum transition amplitude is too low. To fix this, we can directly implement the repeat-until-success scheme proposed in Ref. [10]. In essence, by using two auxiliary states [such as $|j, \pm(m \neq j)\rangle$ ], one can 
define a binary tree of projections that guarantees that each of the four steps in our scheme happens with near certainty.

Besides the transition amplitude, noise and decoherence are also present in our system. Although our reliance on holonomies offers some built-in protection [30], we can add an extra layer of security by realizing that there is a natural link between discrete holonomies and quantum error correction. For clarity, we restrict our discussion to $j=\frac{3}{2}$. Observe that we can view our SCSs as logical qubits: $\left|\frac{3}{2}\right\rangle=$ $\left|\frac{1}{2}\right\rangle^{\otimes 3}=\left|0_{\mathrm{L}}\right\rangle$ and $\left|-\frac{3}{2}\right\rangle=\left|-\frac{1}{2}\right\rangle^{\otimes 3}=\left|1_{\mathrm{L}}\right\rangle$. A bit-flip code [17] can be integrated directly into our scheme if we perform syndrome measurements and corrections after each projection onto a subspace. After completing the repeat-until-success procedure to get the qubit into the correct subspace, we perform error correction measurements to correct any remaining single-qubit bit-flip errors. The syndrome measurements rotate with our states. This means a measurement of, say, $\sigma_{z}^{i} \otimes$ $\sigma_{z}^{j}$ will need to be replaced by $\mathcal{D}_{\mathbf{n}}^{i} \sigma_{z}^{i}\left(\mathcal{D}_{\mathbf{n}}^{i}\right)^{\dagger} \otimes \mathcal{D}_{\mathbf{n}}^{j} \sigma_{z}^{j}\left(\mathcal{D}_{\mathbf{n}}^{j}\right)^{\dagger}=$ $\left(\mathbf{n} \cdot \boldsymbol{\sigma}^{i}\right) \otimes\left(\mathbf{n} \cdot \boldsymbol{\sigma}^{j}\right)$. This is advantageous because we only have to rotate before a gate measurement. Implementing the extra syndrome measurements and the repeat-until-success scheme does not introduce extra rotations. Furthermore, accurate rotations can be achieved experimentally because a rotated measurement of a state is equivalent to a collective rotation of the devices that read out the spin- $\frac{1}{2}$ qubits.

It is also possible to implement the Shor code, which corrects arbitrary errors in single, physical qubits due to decoherence [18]. So now, after the repeat-until-success procedure, we can correct arbitrary remaining single-qubit errors. We define the logical qubits as $\left|0_{\mathrm{L}}\right\rangle=\frac{1}{2 \sqrt{2}}\left(\left|\frac{3}{2}\right\rangle+\left|-\frac{3}{2}\right\rangle\right)^{\otimes 3}$ and $\left|1_{\mathrm{L}}\right\rangle=\frac{1}{2 \sqrt{2}}\left(\left|\frac{3}{2}\right\rangle-\left|-\frac{3}{2}\right\rangle\right)^{\otimes 3}$. Again, the syndrome measurements follow our states' rotation. Unlike the bit-flip code, our logical qubit states are not the same as the SCSs. This means the exact rotation angles, given in Eq. (19), will be different. It is also nontrivial that our new overlap matrices still decompose into a scalar times a unitary matrix. To prove this, we abbreviate $e^{i \theta_{a} J_{y}} e^{-i\left(\phi_{b}-\phi_{a}\right) J_{z}} e^{-i \theta_{b} J_{y}}=U$. Sufficient conditions are that $\left\langle 1_{\mathrm{L}}|U| 0_{\mathrm{L}}\right\rangle=-\left\langle 0_{\mathrm{L}}|U| 1_{\mathrm{L}}\right\rangle^{*}$ and $\left\langle 1_{\mathrm{L}}|U| 1_{\mathrm{L}}\right\rangle=$ $\left\langle 0_{\mathrm{L}}|U| 0_{\mathrm{L}}\right\rangle^{*}$. Since the three groups of physical qubits are rotated by the same unitary $U$ we have that

$$
\begin{aligned}
\left\langle 0_{\mathrm{L}}|U| 1_{\mathrm{L}}\right\rangle & =\left[\left(\left\langle\frac{3}{2}\left|+\left\langle-\frac{3}{2}\right|\right) U\left(\left|\frac{3}{2}\right\rangle-\left|-\frac{3}{2}\right\rangle\right)\right]^{3}\right.\right. \\
& =\left(R-R^{*}-S-S^{*}\right)^{3}, \\
\left\langle 1_{\mathrm{L}}|U| 0_{\mathrm{L}}\right\rangle & =\left(-R+R^{*}+S+S^{*}\right)^{3} \\
& =(-1)^{3}\left[\left(R-R^{*}-S-S^{*}\right)^{*}\right]^{3} \\
& =-\left\langle 0_{\mathrm{L}}|U| 1_{\mathrm{L}}\right\rangle^{*} .
\end{aligned}
$$

A similar calculation proves the diagonal elements. This shows that error correction codes and discrete holonomies can be merged into a single scheme.

We should mention that the repeat-until-success scheme and the syndrome measurements are two separate, distinct steps. It is not sufficient to combine the syndrome measurements and the projection measurements into a single step. This is because the different subspaces into which we project are not close together, so the probability of an error involving more than one qubit is high. These multiqubit errors cannot

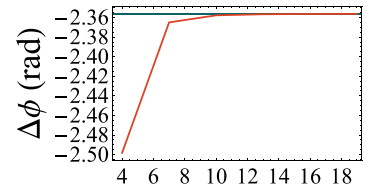

Number of measurements

(a)

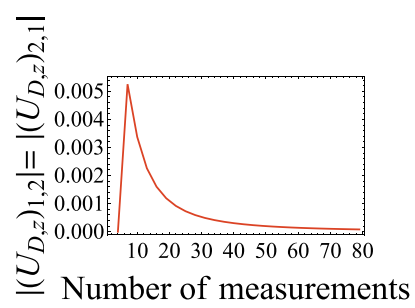

(c)

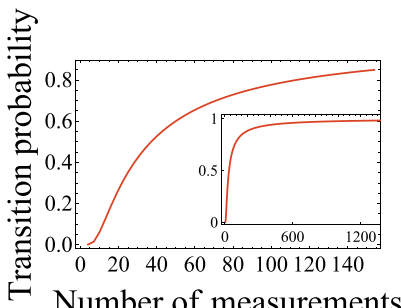

(b)

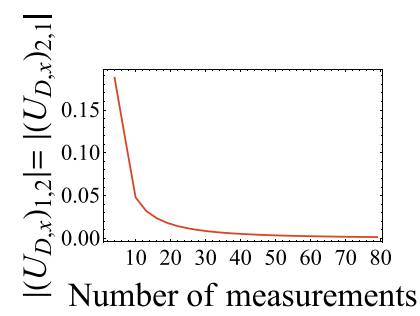

(d)
FIG. 2. The behavior of the discrete holonomy $U_{D}$ for a subspace rotation of $\varphi=\frac{\pi}{4}$ (using a $Z$ gate or an $X$ gate) when the number of measurements is increased. (a) The argument of the relative phase of the diagonal elements of the holonomy for the $Z$ gate. (b) The transition probability for an initial state $\frac{1}{\sqrt{2}}(|j\rangle+|-j\rangle)$ for the $Z$ gate. The inset shows a zoomed-out version of the larger plot. (c) The absolute value of the offdiagonal elements of the holonomy for the $Z$ gate. (d) The absolute value of the off-diagonal elements of the holonomy for the $X$ gate.

be fixed with only the Shor code, which is why we also need the repeat-until-success scheme.

In line with this, it is the combination of the repeat-untilsuccess scheme and the Shor code that makes our scheme fault tolerant, up to errors in the syndrome measurements. Multiqubit errors get fixed by the repeat-until-success scheme, and if any single-qubit errors remain these get fixed by the Shor code. Only if the syndrome measurements of the Shor code go wrong do we get an error. In this sense, our scheme is as fault tolerant as the Shor code.

\section{ZENO LIMIT}

We conclude our discussion on discrete holonomic gates by verifying the continuous-path, or Zeno, limit. It is a well-known result that taking the limit of infinitely many projective measurements "freezes" a quantum system, or drives it through different subspaces with effective certainty [31].

For our choice of SCSs, when we take the limit to dense measurements, we find that we can only implement a phase gate [32]. To get a noncommuting holonomy with the SCSs, necessary for universality, we require a finite number of projections. In passing, we note that if $m= \pm \frac{1}{2}$ instead of $\pm j$ the holonomy would remain non-Abelian in the Zeno limit [16].

We take the Zeno limit for the $\mathcal{D}_{z}(\phi)$ gate. As there is some freedom in what measurement sequence to take, we pick $(0,0) \rightarrow\left(\frac{\pi}{2}, 0\right) \rightarrow\left(\frac{\pi}{2}, \varphi\right) \rightarrow(0,0)$ for simplicity. One can show that we expect a continuous-path holonomy given by $U_{D}=\exp \left(i j \sigma_{z} \int_{0}^{\varphi} \mathrm{d} \phi\right)$. 
Suppose we have $j=\frac{3}{2}$ and rotate our subspace by $\varphi=\frac{\pi}{4}$. In the Zeno limit, our holonomy should be a phase shift with argument $\Delta \phi=-\frac{3 \pi}{4} \approx-2.35$ [33]. If we choose the initial state $|\psi\rangle=\frac{1}{\sqrt{2}}(|j\rangle+|-j\rangle)$ (say), we can also calculate the transition probability. This probability should go to 1 as the number of measurements increases, because of the quantum Zeno effect. Furthermore, the off-diagonal elements of the holonomy $U_{D}$ should tend to zero. This means that increasing the number of measurements to increase the transition amplitude comes with the downside of changing the holonomy. That the off-diagonal matrix elements tend to zero also holds for holonomies that are initially non-Abelian, such as a rotation of $\frac{\pi}{4}$ around the $x$ axis. The argument of the relative phase shift of the diagonal elements should go to $-\frac{3 \pi}{4}$ for $j=\frac{3}{2}$. Numerical calculations confirm this, as shown in Fig. 2.

\section{CONCLUSIONS}

In conclusion, we have demonstrated that discrete holonomic quantum computation can achieve universality. In particular, we have explicitly constructed quantum gates for spin-coherent states, whereby rotation gates were achieved using a sequence of four projective measurements. We have further shown that it is possible to construct an entangling two-qubit gate. These results widen the scope of holonomic gates to include spin-coherent states. Our scheme offers an extra layer of protection as we can readily integrate quantum error correction codes, such as Shor's code. The low transition rate for the projective measurements can be mitigated using a repeat-until-success scheme. This means that, at the cost of more measurements, the error correction code and repeatuntil-success scheme can be combined, though this warrants a more detailed investigation. We also recover previously found results for continuous-path holonomic quantum computation by taking the limit to infinitely many measurements. Our paper demonstrates a deep connection between quantum error correcting codes and discrete holonomies, and highlights the differences between the continuous and discrete realms. The proposed scheme provides a model for measurementbased quantum computation that combines the passive error resilience of holonomic quantum computation and active error correction techniques. Our paper should be viewed as a proof of principle; our idea is not limited to the Shor code. It should be investigated to what extent other quantum codes can be merged with (discrete) holonomic quantum computation. For example, the [[5,1,3]] code [34] could be used instead of the Shor code, or syndrome measurements could be supplemented with flag qubits [35] and gauge qubits [36].

\section{ACKNOWLEDGMENT}

E.S. acknowledges financial support from the Swedish Research Council (VR) through Grant No. 2017-03832.
[1] P. Zanardi and M. Rasetti, Holonomic quantum computation, Phys. Lett. A 264, 94 (1999).

[2] J. Pachos and P. Zanardi, Quantum holonomies for quantum computing, Int. J. Mod. Phys. B 15, 1257 (2001).

[3] L.-M. Duan, J. I. Cirac, and P. Zoller, Geometric manipulation of trapped ions for quantum computation, Science 292, 1695 (2001).

[4] L. Faoro, J. Siewert, and R. Fazio, Non-Abelian Holonomies, Charge Pumping, and Quantum Computation with Josephson Junctions, Phys. Rev. Lett. 90, 028301 (2003).

[5] P. Solinas, P. Zanardi, N. Zanghí, and F. Rossi, Semiconductorbased geometrical quantum gates, Phys. Rev. B 67, 121307(R) (2003).

[6] K. Toyoda, K. Uchida, A. Noguchi, S. Haze, and S. Urabe, Realization of holonomic single-qubit operations, Phys. Rev. A 87, 052307 (2013).

[7] F. Leroux, K. Pandey, R. Rehbi, F. Chevy, C. Miniatura, B. Grémaud, and D. Wilkowski, Non-Abelian adiabatic geometric transformations in a cold strontium gas, Nat. Commun. 9, 3580 (2018).

[8] J. Anandan and A. Pines, Non-Abelian geometric phase from incomplete quantum measurements, Phys. Lett. A 141, 335 (1989).

[9] E. Sjöqvist, D. Kult, and J. Åberg, Manifestations of quantum holonomy in interferometry, Phys. Rev. A 74, 062101 (2006).

[10] D. K. L. Oi, Unitary holonomies by direct degenerate projections, Phys. Rev. A 89, 050102(R) (2014).

[11] J. M. Radcliffe, Some properties of coherent spin state, J. Phys. A: Gen. Phys. 4, 313 (1971).
[12] A. Peres, Quantum Theory: Concepts and Methods (Kluwer, Dordrecht, 1995), pp. 328-329.

[13] R. A. Estrada, E. R. de Azevedo, E. I. Duzzioni, T. J. Bonagamba, and M. H. Y. Moussa, Spin coherent states in NMR quadrupolar system: Experimental and theoretical applications, Eur. Phys. J. D 67, 127 (2013).

[14] T. Chalopin, C. Bouazza, A. Evrard, V. Makhalov, D. Dreon, J. Dalibard, L. A. Sidorenkov, and S. Nascimbene, Quantumenhanced sensing using non-classical spin states of a highly magnetic atom, Nat. Commun. 9, 4955 (2018).

[15] C. Chryssomalakos, E. Guzmán-González, and E. SerranoEnsástiga, Geometry of spin coherent states, J. Phys. A: Math. Theor. 51, 165202 (2018).

[16] A. Zee, Non-Abelian gauge structures in nuclear quadrupole resonance, Phys. Rev. A 38, 1 (1988).

[17] A. Peres, Reversible logic and quantum computers, Phys. Rev. A 32, 3266 (1985).

[18] P. W. Shor, Scheme for reducing decoherence in quantum computer memory, Phys. Rev. A 52, R2493 (1995).

[19] R. Raussendorf and H. J. Briegel, A One-Way Quantum Computer, Phys. Rev. Lett. 86, 5188 (2001).

[20] M. A. Nielsen, Quantum computation by measurement and quantum memory, Phys. Lett. A 308, 96 (2003).

[21] C. A. Mead, "Block diagonalization" as generalization of Pancharatnam-Berry phase relation for multidimensional spaces, Phys. Rev. A 44, 1473 (1991).

[22] D. Kult, J. Åberg, and E. Sjöqvist, Noncyclic geometric changes of quantum states, Phys. Rev. A 74, 022106 (2006). 
[23] Note that, besides mathematically, we should also physically interpret our state $|j\rangle$ to be a composite system of spin- $\frac{1}{2}$ particles. One should thus not think of $|j\rangle$ as (say) a spin- $j$ atom but as a composite of spin- $\frac{1}{2}$ qubits.

[24] We stress that this requires $j$ to be a half-odd integer. If $j$ is an integer then $\kappa^{-1}$ ceases to be a number and becomes a positive semidefinite matrix instead, which is considerably more complicated when combined with the other overlap matrices in the sequence [9].

[25] For $q=2$, the holonomy $U_{D}$ reduces to the identity transformation as $\left(\mathcal{F}_{1} \mid \mathcal{F}_{2}\right)\left(\mathcal{F}_{2} \mid \mathcal{F}_{1}\right)=\left|\left(\mathcal{F}_{1} \mid \mathcal{F}_{2}\right)\right|^{2}>0$

[26] In the continuous-path case one can pick coordinates that remove one (but not both) of the singularities. The two patches that cover the system are related by a gauge transformation [16].

[27] W. K. Wootters, Entanglement of Formation of an Arbitrary State of Two Qubits, Phys. Rev. Lett. 80, 2245 (1998).

[28] M. J. Bremner, C. M. Dawson, J. L. Dodd, A. Gilchrist, A. W. Harrow, D. Mortimer, M. A. Nielsen, and T. J. Osborne, Practical Scheme for Quantum Computation with Any Two-Qubit Entangling Gate, Phys. Rev. Lett. 89, 247902 (2002).

[29] B. Giles and P. Selinger, Exact synthesis of multiqubit Clifford $+T$ circuits, Phys. Rev. A 87, 032332 (2013).

[30] I. Fuentes-Guridi, F. Girelli, and E. Livine, Holonomic Quantum Computation in the Presence of Decoherence, Phys. Rev. Lett. 94, 020503 (2005).
[31] Y. Aharonov and M. Vardi, Meaning of an individual "Feynman path," Phys. Rev. D 21, 2235 (1980).

[32] To see why, let us divide the total angles into $N$ steps, $\delta \theta=\theta / N$ and $\delta \phi=\phi / N$, and take the limit $N \rightarrow \infty$. Then, one of the overlap matrices $\left(\mathcal{F}\left(\theta_{a}+\delta \theta, \phi_{a}+\delta \phi\right) \mid \mathcal{F}\left(\theta_{a}, \phi_{a}\right)\right)$ contains (up to and including first order) $e^{i\left(\theta_{a}+\delta \theta\right) J_{y}} e^{i\left(\phi_{a}+\delta \phi\right) J_{z}} e^{-i \phi_{a} J_{z}} e^{-i \theta_{a} J_{y}}=$ $1+i \delta \theta J_{y}+i \delta \phi\left[-\sin \left(\theta_{a}\right) J_{x}+\cos \left(\theta_{a}\right) J_{z}\right]$. Since $j \geqslant 1$ the offdiagonal elements of the overlap matrix vanish and the diagonal elements become $1 \pm i j \cos \left(\theta_{a}\right) \delta \phi$. Therefore, in the limit $N \rightarrow$ $\infty$ the total holonomy can only be a phase gate.

[33] The appropriate way to take the Zeno limit is to increase the number of measurements along geodesics connecting the initial points. In taking the limit it does not matter from which $\phi$ coordinate we approach the $(0,0)$ subspace, as all subspaces at the north pole are connected by a $U(1)$ gauge transformation and projectors are gauge invariant.

[34] R. Laflamme, C. Miquel, J. P. Paz, and W. H. Zurek, Perfect Quantum Error Correcting Code, Phys. Rev. Lett. 77, 198 (1996).

[35] R. Chao and B. W. Reichardt, Quantum Error Correction with Only Two Extra Qubits, Phys. Rev. Lett. 121, 050502 (2018).

[36] E. T. Campbell, B. M. Terhal, and C. Vuillot, Roads towards fault-tolerant universal quantum computation, Nature (London) 549, 172 (2017). 